\title{
Smallest Organic Tetracation in Gas Phase: Stability of Multiply Charged Diiodoacetylene Produced in Intense Femtosecond Laser Fields
}

\author{
Supporting Information
}

Takashi Kawaguchi, Kosei Kitagawa, Kazuo Toyota, * Masatoshi Kozaki, Keiji Okada, ${ }^{\dagger}$ Nobuaki Nakashima, Tomoyuki Yatsuhashi*

Department of Chemistry, Graduate School of Science, Osaka City University, 3-3-138 Sugimoto, Sumiyoshi, Osaka 558-8585 Japan 

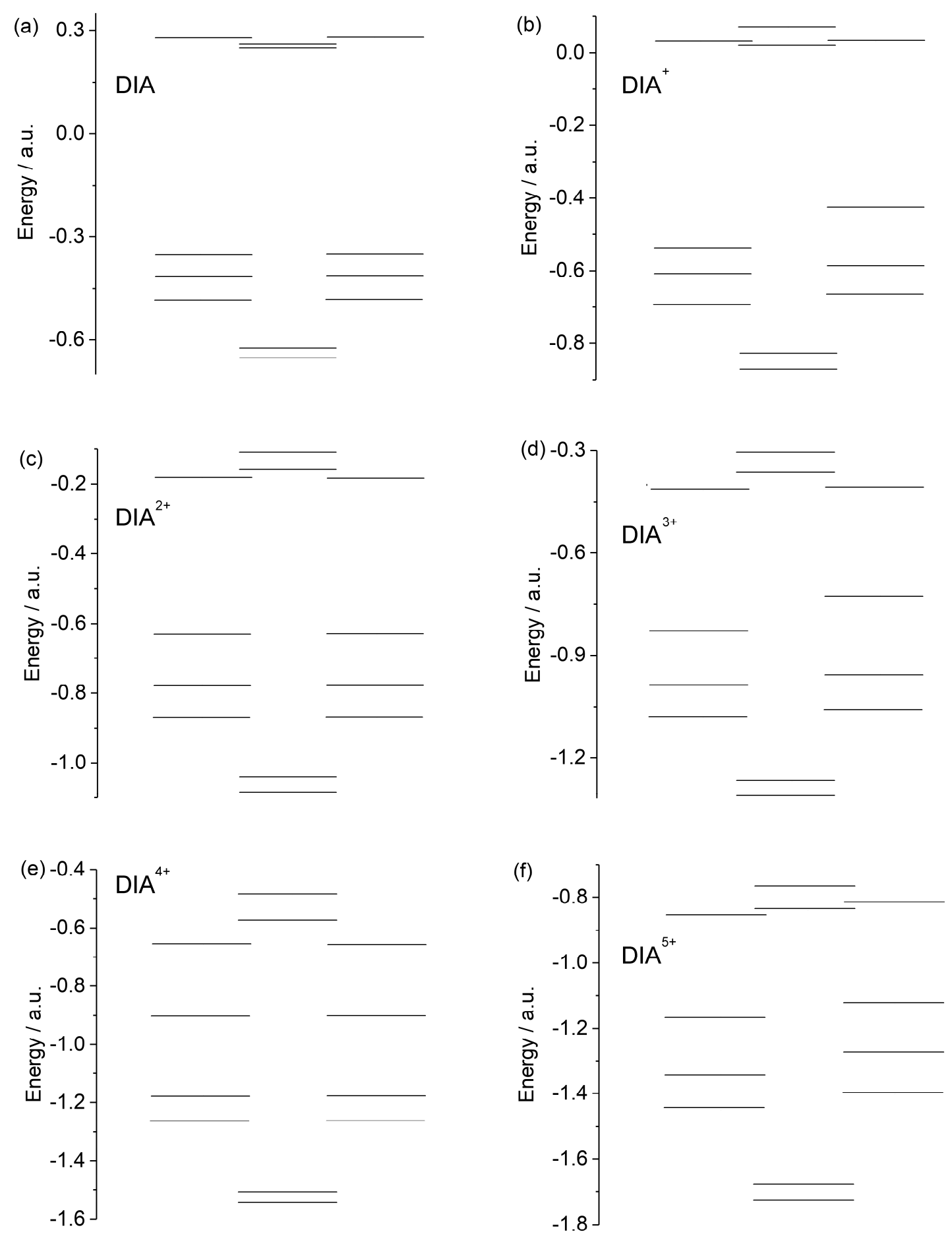

Figure S1. Molecular orbitals of $\mathrm{DIA}^{z^{+}}(z=0-4)$ at equilibrium configuration calculated by CASSCF: (a) DIA, (b) DIA ${ }^{+}$, (c) $\mathrm{DIA}^{2+}$, (d) $\mathrm{DIA}^{3+}$, (e) $\mathrm{DIA}^{4+}$. (f) The molecular orbitals of DIA $^{5+}$ calculated by CASSCF method for the optimized structure obtained by DFT are also shown. 


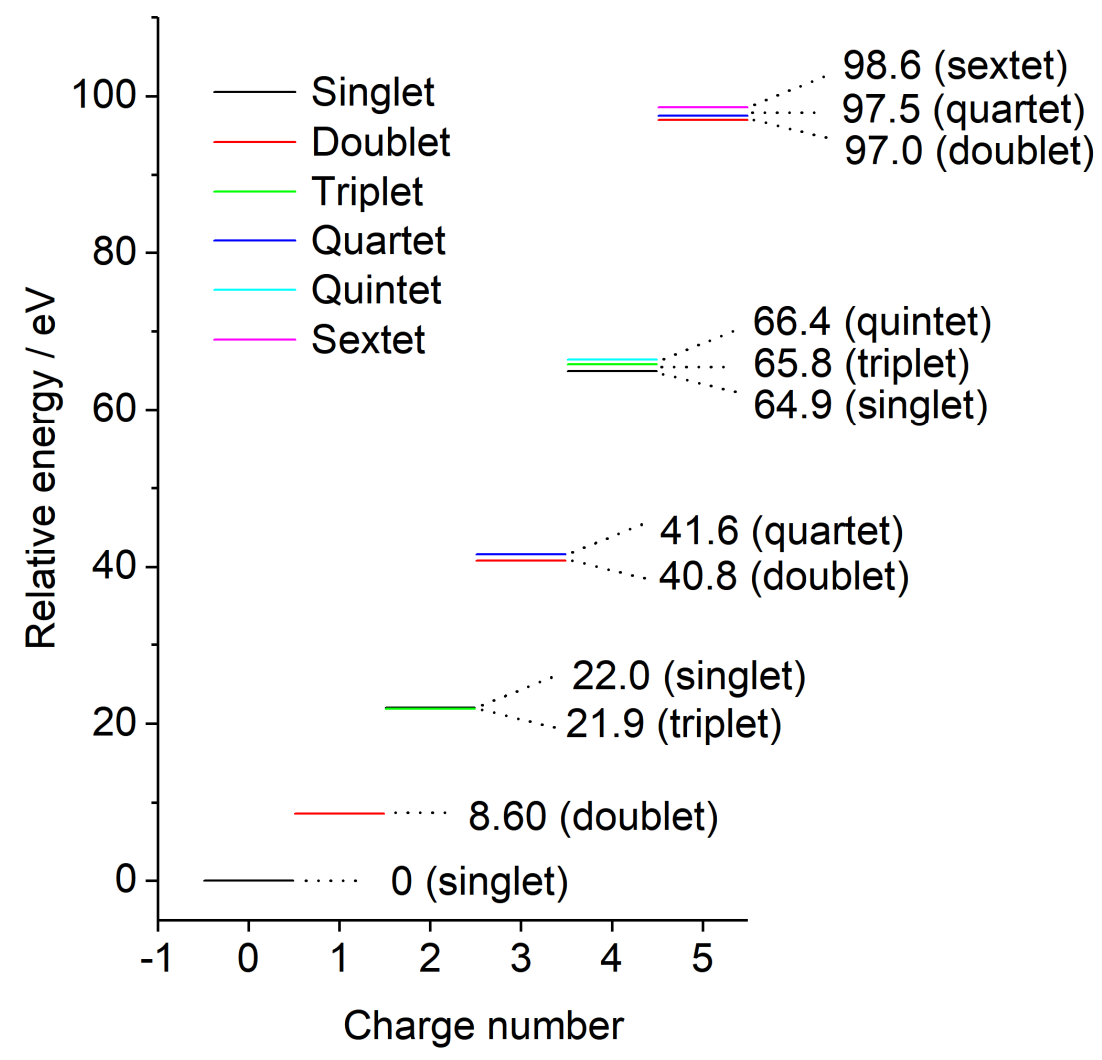

Figure S2. Energy level of DIA $z^{z^{+}}(z=1-4)$ at equilibrium configuration calculated by CASSCF. The energy levels of DIA ${ }^{5+}$ were calculated by CASSCF method for the fixed molecular structure optimized by DFT calculations. 